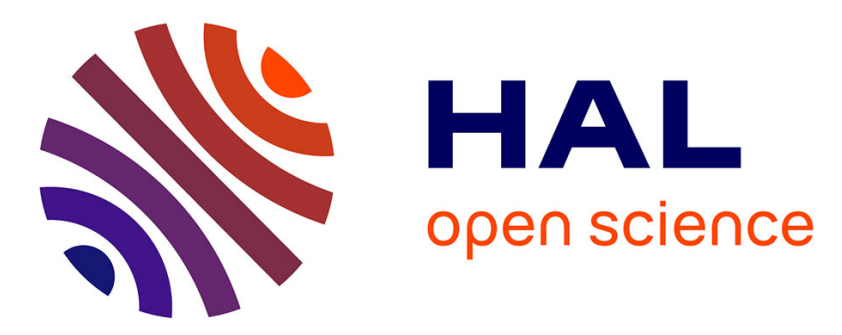

\title{
Recursive identification of an arterial baroreflex model for the evaluation of cardiovascular autonomic modulation
}

Virginie Le Rolle, Alain Beuchée, Jean-Paul Praud, Nathalie Samson, Patrick Pladys, Alfredo I Hernández

\section{To cite this version:}

Virginie Le Rolle, Alain Beuchée, Jean-Paul Praud, Nathalie Samson, Patrick Pladys, et al.. Recursive identification of an arterial baroreflex model for the evaluation of cardiovascular autonomic modulation. Computers in Biology and Medicine, 2015, 66, pp.287-294. 10.1016/j.compbiomed.2015.09.013 . hal-01262697

\section{HAL Id: hal-01262697 https://hal-univ-rennes1.archives-ouvertes.fr/hal-01262697}

Submitted on 11 Feb 2016

HAL is a multi-disciplinary open access archive for the deposit and dissemination of scientific research documents, whether they are published or not. The documents may come from teaching and research institutions in France or abroad, or from public or private research centers.
L'archive ouverte pluridisciplinaire HAL, est destinée au dépôt et à la diffusion de documents scientifiques de niveau recherche, publiés ou non, émanant des établissements d'enseignement et de recherche français ou étrangers, des laboratoires publics ou privés. 


\title{
Recursive identification of an arterial baroreflex model for the evaluation of cardiovascular autonomic modulation
}

\author{
Virginie Le Rolle ${ }^{1,2}$, Alain Beuchee ${ }^{1,2,3}$, Jean-Paul Praud ${ }^{4}$, Nathalie \\ Samson $^{4}$, Patrick Pladys ${ }^{1,2,3}$, Alfredo I. Hernández ${ }^{1,2}$ \\ ${ }^{1}$ INSERM, U1099, Rennes, F-35000, France \\ 2 Université de Rennes 1, LTSI, Rennes, F-35000, France \\ ${ }^{3}$ CHU Rennes, Pole de pdiatrie mdico-chirurgicale et gntique clinique-Service de \\ pdiatrie, Rennes, F-35000, France \\ ${ }^{4}$ Department of Pediatrics, University of Sherbrooke, J1H5N4, QC-Canada \\ Corresponding author : Virginie Le Rolle \\ LTSI, Campus de Beaulieu, Université de Rennes 1, 263 Avenue du General \\ Leclerc \\ CS 74205 \\ 35042 Rennes Cedex, France \\ Tel.: +33223235903 \\ Fax: +33223236917 \\ virginie.lerolle@univ-rennes1.fr
}

\begin{abstract}
The evaluation of the time-varying vagal and sympathetic contributions to heart rate remains a challenging task because the observability of the baroreflex is generally limited and the time-varying properties are difficult to take into account, especially in non-stationnary conditions. The objective is to propose a model-based approach to estimate the autonomic modulation during a pharmacological challenge.

A recursive parameter identification method is proposed and applied to a mathematical model of the baroreflex, in order to estimate the time-varying vagal and sympathetic contributions to heart rate modulation during autonomic maneuvers. The model-based method was evaluated with data from five newborn lambs, which were acquired during injection of vasodilator and vasoconstrictor drugs, on normal conditions and under beta-blockers, so as
\end{abstract}


to quantify the effect of the pharmacological sympathetic blockade on the estimated parameters.

After parameter identification, results show a close match between experimental and simulated signals for the five lambs, as the mean relative root mean squared error is equal to $0.0026(+/-0.003)$. The error, between simulated and experimental signals, is significantly reduced compared to a batch identification of parameters. The model-based estimation of vagal and sympathetic contributions were consistent with physiological knowledge and, as expected, it was possible to observe an alteration of the sympathetic response under beta-blockers. The simulated vagal modulation illustrates a response similar to traditional heart rate variability markers during the pharmacological maneuver. The model-based method, proposed in the paper, highlights the advantages of using a recursive identification method for the estimation of vagal and sympathetic modulation.

Keywords: keywords : Model-based method, Autonomic nervous system, Parameter identification, Physiological modelling.

\section{Introduction}

During the first days of life, newborns may be exposed to various life threatening events, such as intracranial hypertension, neonatal septic shock or streptococcus b infection. These events induce physiological challenges that trigger protective autonomic autoresuscitation reflexes and the evolution of the newborn state directly depends on the responses of both vagal and sympathetic nervous activities. Monitoring the status of infants in the Neonatal Intensive Care Unit (NICU) provides data of physiological variables (arterial pressure, heart rate,...). Although the prognosis of the newborn will depend on the maturity of his or her autonomic nervous system, information about autonomic adaptation is difficult to access. As a consequence, robust tools are required to evaluate time-varying evolutions of vagal and sympathetic responses during newborn monitoring in NICU. In fact, evaluating sympathetic and parasympathetic tones will give precious information for the diagnosis and the choice of the most appropriate treatment.

Informations about the autonomic status are usually provided by Heart Rate Variability (HRV) analysis, based time or frequency domaine indicators. In particular, the high-frequency (HF) components of the power spectrum of the HRV signal have been widely used as an indicator of the parasympathetic 
modulation and the low-frequency components (LF), are considered as a mix of sympathetic and parasympathetic modulations. Time-frequency analyses of heart rate (HR) series have also been applied to estimate these frequencydomain markers in non-stationary conditions [1]. Another approach is based on the application of blind source separation methods. Vetter et al [2] have proposed a method based on independent component analysis (ICA) for the estimation of the sympathetic, parasympathetic and respiratory activities as three different, synchronous time-varying sources. Previous work of our team extended this approach to an asynchronous processing of the observables [3].

However, it is difficult to interpret the results of most of these methods in physiological terms, since no a priori knowledge about the underlying physiology is integrated into the analysis. For instance, it is difficult to separate the sympathetic and parasympathetic contributions to the LF marker or the mechanical respiratory effect from the parasympathetic modulation of the HF marker [4]. As a consequence, complimentary approaches are needed in order to improve of the evaluation of sympathetic and parasympathetic modulation and the interpretation of clinical data.

Yet another approach consists in the integration of physiological knowledge into the analysis, by means of physiological models, integrating not only the effect of ANS on HR, but also other physiological parameters. Several models of the baroreflex, based on adult data, have been already proposed. Those based on autoregressive representations are particularly adapted to study interactions between RR interval, arterial pressure and respiration $[5,6,7]$. Other models include an explicit representation of vagal and sympathetic nervous systems $[8,9]$. Transfer functions are largely used to represent the components of the baroreflex loop [10, 11, 12]. Most of them take into account the non-linear behavior of the autonomic modulation [13, 14]. Some models have been employed to reproduce non-stationnary clinical tests, such as the orthostatic stress $[15,16,17]$ and Valsalva maneuvers $[11,18]$. However, most of these models rely on population-based parameters (not patient-specific parameters) or neglect their time-varying nature.

In this work, we propose an original approach, based on a model of the baroreflex function, introducing: i) a phase of subject-specific model parameter identification and ii) a phase of estimation of the time-varying sympathetic and parasympathetic modulations, through the use of a recursive evolutionary algorithm. The proposed approach was evaluated with cardiorespiratory data acquired from five newborn lambs, during non-stationary conditions, provoked by the injection of a vasodilator and a vasoconstrictor 
drug, in normal conditions and during sympathetic blockade. Results from the proposed approach were compared to traditional autonomic markers, extracted from time-frequency HRV analysis.

\section{Methods}

\subsection{Data and experimental protocol}

Experiments were performed on lambs aged 4-5 days. All lambs were full-term at birth and housed with their mother. The protocol was approved by the Committee for Animal Care and Experimentation of the University of Sherbrooke, Canada. Surgery was performed two days before the experiment under general anesthesia following the procedure detailed by Duvareille et al. [19] for catheter implanting. Systemic arterial pressure (AP) was obtained from the brachial catheter using a pressure transducer (Trantec model 60-800, American Edwards Laboratories, Santa Anna, CA, USA) and pressure monitor (model 78342A Hewlett Packard, Waltham, MA, USA). Respiratory thoraco-abdominal movements were monitored with a respiratory inductance plethysmography (RIP) system (Respitrace; NIMS Inc., Miami, FL, USA). Two electrocardiogram (ECG) channels were acquired using a couple of ECG100 modules (Biopac Systems, Inc. Santa Barbara, CA, USA). All signals (ECG, AP and respiration) were sampled at $1000 \mathrm{~Hz}$ and recorded on a PC, using the MP100A data acquisition system and Acknowledge 3.7.3 software (Biopac Systems Inc. Santa Barbara, CA, USA).

Lambs were non-sedated and, throughout the recordings, were comfortably positioned in a sling with loose restraints and monitored with the abovementioned recording system. Ambient temperature was $22^{\circ} \mathrm{C}$. An observer was always present in the laboratory to note all events. The sequence of experimentations started with a 3 min recording in basal conditions, while during behaviorally-defined quiet sleep, followed by a continuous perfusion of nitroprusside sodium $\left(12 \mu \mathrm{g} \cdot \mathrm{kg}^{-1} \cdot \mathrm{min}^{-1}\right)$ for 360 seconds.

In the standardization process, we aim to maintain the state of alertness (quiet sleep), which is quiet frequent but with short duration in full-term lamb. The 3-min duration is a good compromise between sufficient duration for the analysis and the preservation of quiet sleep. Subsequently, after a 30 min period of recovery, a bolus injection of nitroprusside $\left(20 \mu \mathrm{g} \cdot \mathrm{kg}^{-1}\right)$ was administrated and was followed, after 120 seconds, by a bolus injection of phenylephrine $\left(4 \mu \mathrm{g} \cdot \mathrm{kg}^{-1}\right)$ ( fig. 1). The same sequence of experimentations was repeated another day, starting 5 minutes after the intravenous bolus 

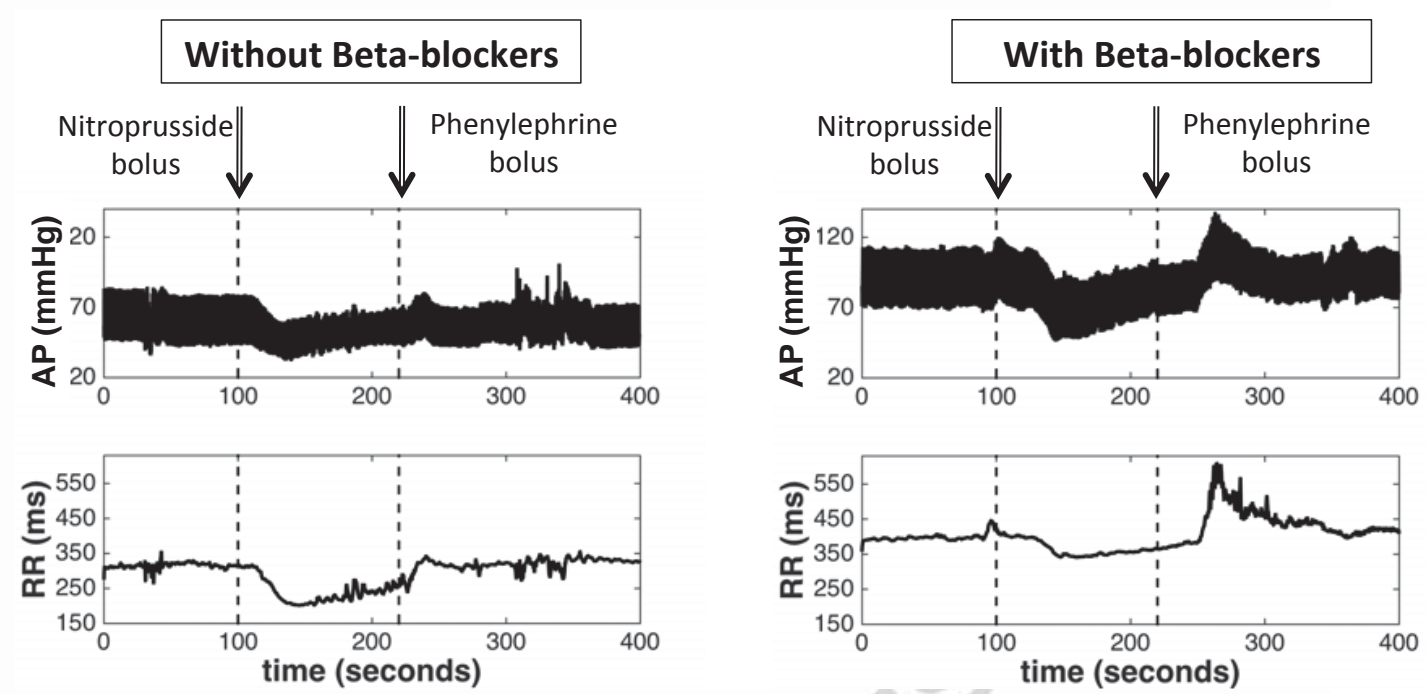

Figure 1: Example of signals acquired with and without beta-blockers on two days. Arterial pressure $(\mathrm{mmHg})$ and $\mathrm{RR}(\mathrm{ms})$ obtained during the perfusion of nitroprusside and after the bolus of phenylephrine.

administration of metoprolol $\left(1 \mathrm{mg} \cdot \mathrm{kg}^{-1}\right)$ repeated each 30 mins. The metoprolol blocks the action of the sympathetic nervous system. The days, with and without beta-blockers, were randomized in order to minimize the residual effect of metoprolol. At the end of the experimentation, the beta-blockers administration is combined to an injection of atropine $\left(200 \mu \mathrm{g} \cdot \mathrm{kg}^{-1}\right)$ in order to obtain the intrinsic heart rate.

\subsection{Baroreflex Model}

The baroreflex model is represented in fig. 2. It includes the baroreceptors and afferent pathways, the cardiovascular control centers and the efferent pathways (including the vagal and sympathetic branches). The structure is based on our previous baroreflex model, which was tested on one lamb signal in [20] and was applied to the analysis of orthostatic stress in [17].

The baroreceptor (B) input is AP and its dynamical properties are represented by a first-order filter, whose gain and time constant are denoted $K_{B}$ and $T_{B}$, respectively. The derivative terme, attributed to baroreceptor dynamics, was neglected in this work in order to limit the number of parameters for the identification step. The cardiovascular control centers are represented by sigmoidal functions and two delays $\left(D_{V}\right.$ and $D_{S}$ are respectively the sym- 


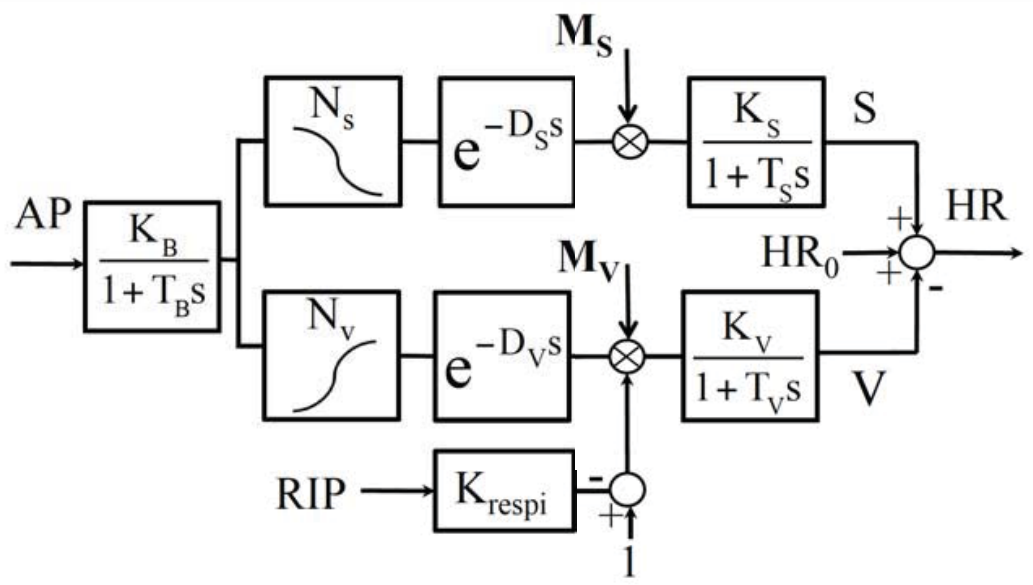

Figure 2: Block diagram of baroreflex control of AP. Baroreceptors are represented by a first-order filter $\left(K_{B}\right.$ and $\left.T_{B}\right)$. Vagal and sympathetic pathways are both modelled by normalization functions $\left(N_{V}\right.$ and $\left.N_{S}\right)$, delays $\left(D_{V}\right.$ and $\left.D_{S}\right)$ and first-order filters characterized by gains $\left(K_{V}\right.$ and $\left.K_{S}\right)$ and time constants $\left(T_{V}\right.$ and $\left.T_{S}\right)$. Time-varying variables $M_{V}(t)$ and $M_{S}(t)$ modulates each nervous pathway. Intrinsic heart rate $\left(H R_{0}\right)$, contributions from sympathetic $(\mathrm{S})$ and vagal $(\mathrm{V})$ branches are summed to obtained HR.

pathetic and parasympathetic delays). The efferent pathways are composed of two first-order filters characterized by a gain $\left(K_{V}\right.$ and $K_{S}$ for the vagal and the sympathetic gains respectively) and a time constant $\left(T_{V}\right.$ and $\left.T_{S}\right)$. This description is similar to other model representations $[10,8,9]$. This kind of formalism allows for the representation of the global neurotransmitter dynamics for a particular efferent pathway and the description of the different time responses of the sympathetic and the parasympathetic branches. Normalization and saturation effects are represented by sigmoidal input-output relationship [11]:

$$
N_{x}=a_{x}+\frac{b_{x}}{e^{\lambda_{x}\left(F_{b}-M_{x, 0}\right)}+1}
$$

where the generic index $\mathrm{x} \in(\mathrm{v}, \mathrm{s})$ stands for the vagal and sympathetic pathways, $F_{B}$ is the baroreceptor output, and the parameters $a_{x}, b_{x}, \lambda_{x}$ and $M_{x, 0}$ are used to adjust the sigmoidal shape.

The main originality of the model is to propose specific variables to describe the short-term modulation of both nervous pathways. The vagal and sympathetic activities are modulated by two time-varying variables $M_{V}(t)$ and $M_{S}(t)$ which represent the influence of different brain structures on va- 
gal and sympathetic pathways. It is important to understand that these two time-varying variables $M_{V}(t)$ and $M_{S}(t)$ aggregate all the influences, which are not due to AP variations. It notably includes the impact of the closedloop structure of the baroreflex [21], the chemoreflex or central influences. This representation allows us to differentiate the part of the vagal and sympathetic nerve traffic that could be attributed to AP variation from other influences.

The vagal activity is modulated by $M_{V}(t)$ and by the lung volume variations, assessed by the sum signal of the uncalibrated respiratory inductance plethysmography (RIP) rescaled in a specific range $(0,1)$. The influence of respiration on HR is modeled in a simple manner [9] by a vagal inhibition during inspiration. The inhibition of vagal activation is implemented as a multiplication by $1-K_{\text {respi }}$.RIP. In this model, two major elements differentiate the sympathetic and vagal activities : 1) the direction of the sigmoidal input-output relationship 2) the differences between vagal and sympathetic time constants $\left(T_{V}\right.$ and $\left.T_{S}\right)$ and delays $\left(D_{V}\right.$ and $\left.D_{S}\right)$. The differentiation between vagal and sympathetic modulations is also attributed to the identification algorithm presented in the next section.

The sinus node response to vagal and sympathetic modulation could be represented by a three-dimensional response surface[22]. However, in order to reduce the number of parameters for the identification step, the continuous output signal of the heart rate regulation model (HR) is obtained by adding the contributions from the sympathetic $(\mathrm{S})$ and vagal $(\mathrm{V})$ branches and a basal (intrinsic) heart rate $\left(H R_{0}\right)$ :

$$
H R=H R_{0}+S-V
$$

The proposed model includes several parameters that should be identified in order to be adapted to the neonatal period and to be able to reproduce signals acquired from a specific lamb. The identification method is presented in the following section.

\subsection{Identification method}

\subsubsection{General framework of the parameters identification.}

The identification process was performed using the experimental AP and RIP as inputs to the baroreflex model. The simulated RR interval signal, which is calculated as the inverse of HR, is compared to the experimental $\mathrm{RR}$ using the error functions described in this section. 


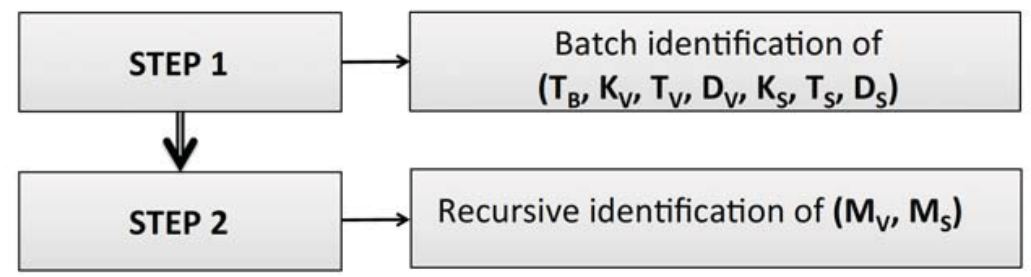

Figure 3: Two steps of the identification process. The constant parameters $\left(T_{B}, K_{V}\right.$, $\left.T_{V}, D_{V}, K_{S}, T_{S}, D_{S}\right)$ are firstly identified by minimizing the error function $\epsilon_{b}$ using evolutionary algorithms. Then, $\left(M_{V}, M_{S}\right)$ are identified recursively by minimizing the error function $\epsilon_{r}$.

One of the main difficulties here is to take into account the different dynamics associated with each parameter. Parameters $T_{B}, K_{V}, T_{V}, D_{V}, K_{S}$, $T_{S}, D_{S}$, which show slower dynamics with respect to beat-to-beat autonomic modulations, were assumed to be constant during the time of the autonomic maneuvers. On the other hand, modulations of vagal and sympathetic activities $\left(M_{V}(t)\right.$ and $\left.M_{S}(t)\right)$ are assumed to present rapid dynamics, which may significantly change during the autonomic maneuver, justifying the timevarying representation proposed in this work. In order to take into account the differences between each kind of variables, the identification procedure was composed of two steps ( fig. 3):

- Step 1: Batch identification of constant parameters $\left(T_{B}, K_{V}, T_{V}, D_{V}\right.$, $\left.K_{S}, T_{S}, D_{S}\right)$ on the complete $\mathrm{RR}$ signal, which total duration is referred as $T_{t o t}$;

- Step 2: Recursive identification of time-varying variables $\left(M_{V}, M_{S}\right)$.

These two steps are associated with errors functions, which are minimized using evolutionary algorithms (EA), as in our previous works [17, 23, 20]. EA are stochastic search methods, inspired by the theories of evolution and natural selection, which can be employed to find an optimal configuration for a given system [24].

\subsubsection{Batch identification of constant parameters}

Concerning the first step ( fig. 4), uniform distribution bounded by feasibility intervals was defined to create the initial population of the EA for each parameter: $(0.01,1)$ for $T_{B}(\mathrm{~s}),(0.01,1.5)$ for $T_{V}(\mathrm{~s}),(0.1,0.5)$ for $D_{V}(\mathrm{~s})$, 


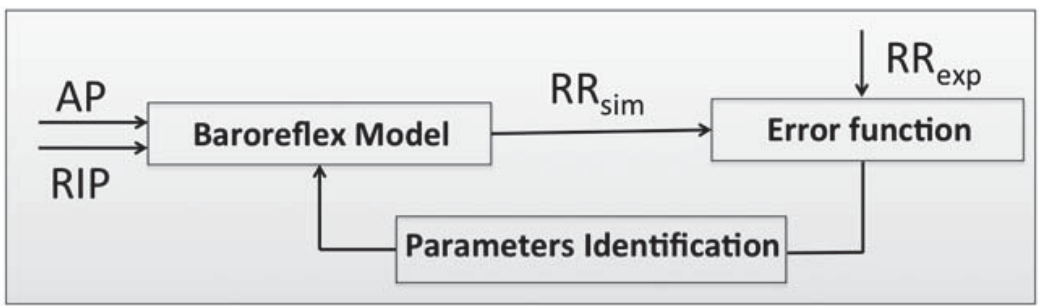

Figure 4: General diagram of the batch identification step. The constant parameters $\left(T_{B}\right.$, $\left.K_{V}, T_{V}, D_{V}, K_{S}, T_{S}, D_{S}\right)$ are first identified by minimizing the error function $\epsilon_{b}$. The experimental signals, AP and RIP, are the input of the baroreflex model, which produces a simulated RR. The error function, between simulated $R R_{\text {sim }}$ and experimental $R R_{\text {exp }}$, is minimized using EA.

$(0.6,360)$ for $K_{V}(\mathrm{bpm}),(5,20)$ for $T_{S}(\mathrm{~s}),(2,6)$ for $D_{S}(\mathrm{~s}),(0.6,360)$ for $K_{S}(\mathrm{bpm})$. These intervals were defined by taking into consideration previously published parameter values $[10,11,9]$ and are large enough to assure an accurate research of parameters values. During the batch identification, an error function $\epsilon_{b}$ was defined on the whole duration of the experimental signal:

$$
\epsilon_{b}=\sum_{t_{e}=0}^{T_{t o t}}\left|R R_{\text {sim }}\left(t_{e}\right)-R R_{e x p}\left(t_{e}\right)\right|
$$

where $t_{e}$ corresponds to the time elapsed since the beginning of nitroprusside injection and $T_{t o t}$ is the length of the experimental signal equal to 200 seconds. The constant parameters, determined from the first step of the identification, are used in the baroreflex model in order to perform the recursive identification of the time-varying variables $\left(M_{V}, M_{S}\right)$.

\subsubsection{Recursive identification of time-varying variables}

The second step ( fig. 5) is based on a recursive parameter identification procedure. At each step $i$ of the algorithm, parameters are identified on a given time interval $\left(T_{I}\right)$, which duration is largely inferior to the total duration of the experiments $\left(T_{t o t}\right)$ such as: $T_{I}<<T_{t o t}$. The following error function is thus minimized: 


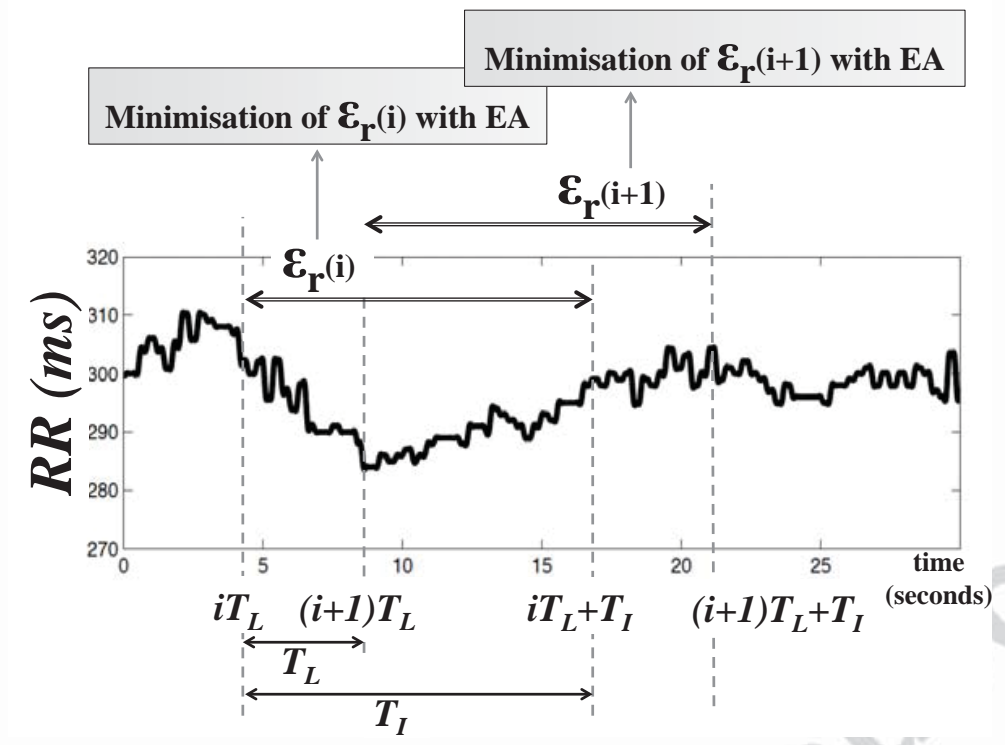

Figure 5: Diagram illustrating the recursive identification algorithm. At the step i, the error function $\epsilon_{r}(\mathrm{i})$ is calculated on the identification interval $T_{I}$ and minimized using EA. At the step $\mathrm{i}+1$, the identification interval is shifted of $T_{L}$ which is the overlap time between each interval, and $\epsilon_{r}(\mathrm{i}+1)$ is also minimized using EA. $T_{I}$ and $T_{L}$ are respectively equal to the sympathetic and vagal time constant $\left(T_{S}\right.$ and $\left.T_{V}\right)$. The longest interval $T_{I}$ is used to identify the low frequency component of the signal whereas the shortest interval $T_{L}$ capture the high frequency compound.

$\epsilon_{r}(i)=\sum_{t_{e}=i T_{L}}^{(i+1) T_{L}}\left|R R_{\text {sim }}\left(t_{e}\right)-R R_{\text {exp }}\left(t_{e}\right)\right|+\sum_{t_{e}=i T_{L}}^{i T_{L}+T_{I}}\left|R R_{\text {sim }}\left(t_{e}\right)-R R_{\text {exp }}\left(t_{e}\right)\right|, i \in[0, \ldots, N]$

where $t_{e}$ corresponds to the time elapsed since the onset of the identification period, $T_{L}$ is the overlap time between each interval and $\mathrm{N}$ is the number of identification intervals, which is equal to the integer part of $T_{t o t} / T_{L}$. The overlap time duration $T_{L}$ was chosen equal to the vagal time constant $\left(T_{V}\right)$ to capture rapid events due to the vagal response. The identification interval $T_{I}$ is equal to the sympathetic time constant $\left(T_{S}\right)$ in order to take into account slow variations.

This error function is minimized on each interval $i$ using EA. Concerning the first interval, a set of random initial solutions was used to create the initial population. For the following intervals, the initial population was 
set equal to the population obtained from interval i-1 considering that the parameter variation between intervals is limited. Although this approach of attribution of initial populations limits the parameters changes, a mutation operator with probability $p_{m}=0.2$ helps the process to explore the entire search space and prevent from convergence to a local minimum.

\subsection{Estimation of the vagal modulation from time-frequency domain methods}

Although, HRV analysis is a subject of controversy [4], the most common autonomic markers are extracted from frequency domain methods [25]. Two major components of the HRV spectrum have been considered: Low Frequency (LF: 0.02-0.25 Hz), and High Frequency (HF: 0.25-2 Hz). These frequency bands have been adapted to the newborn lamb, as proposed in our previous work [26].

The HF component of the RR series is widely believed to reflect cardiac efferent parasympathetic modulation [25]. The interpretation of the LF component is more complex because it includes both sympathetic and vagal influences $[27,28,29]$. Usually spectral approaches require the signals to be stationary, which is inappropriate for studying non-stationary processes induced by pharmacological stimulations. To overcome these limitations, a time-frequency analysis is used to evaluate spectral characteristics. In this paper, a smoothed pseudo-Wigner-Ville distribution (SPWVD) was used because it has proved its usefulness for the analysis of cardiovascular signals $[1]:$

$$
C_{x}(t, f)=W_{x}(t, f) \otimes \phi_{x}(t, f)
$$

where $C_{x}$ is a Cohen's class of quadratic time-frequency distributions, $\otimes$ represents the convolution on $\mathrm{t}$ and $\mathrm{f}, \phi_{x}$ is a Cohen kernel function, which parameters were defined from $[30,1]$ and $W_{x}$ is the Wigner-Ville distribution:

$$
W_{x}(t, f)=\int_{-\infty}^{\infty} x(t+\tau / 2) x^{*}(t-\tau / 2) e^{-2 \pi \tau f} \mathrm{~d} \tau
$$

where $*$ corresponds to the complex conjugation. The high frequency power of $\mathrm{RR}(\mathrm{HF}$ ) was computed in the 0.25 to $2 \mathrm{~Hz}$ band in order to reconstruct the evolution of $\mathrm{HF}$ component with respect to time.

$$
E_{H F}(t)=\int_{H F} C_{x}(t, f) \mathrm{d} f
$$




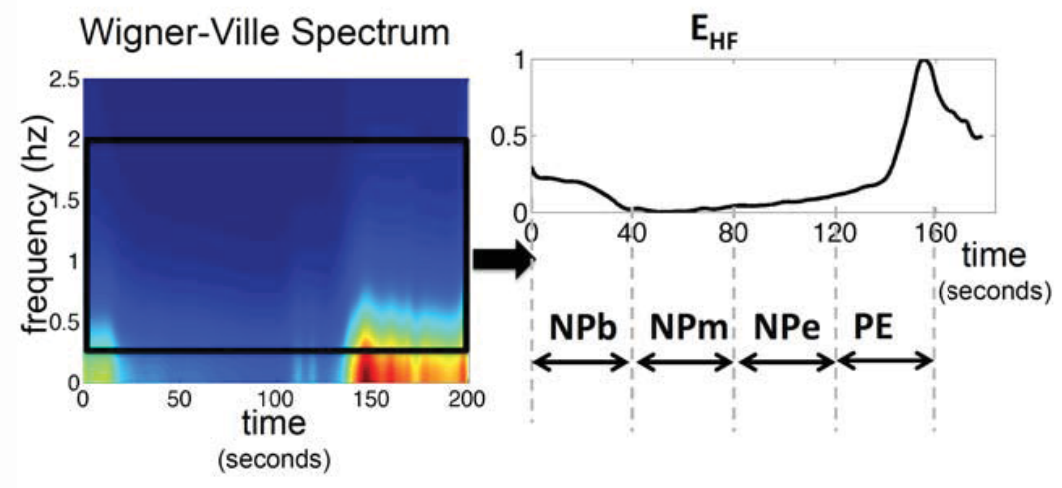

Figure 6: Wigner-Ville time-frequency representations obtained from the RR signals of lamb 1, without sympathetic blockade. Four periods of 40 seconds were defined: $\mathrm{NPb}$ (Nitroprusside beginning) for [0s,40s], NPm (Nitroprusside middle) for [40s,80s], NPe (Nitroprusside end) for [80s,120s] and PE (Phenylephrine) for [120s,160s].

Four periods of 40 seconds were defined in order to capture the variations of $\mathrm{HF}$ during the pharmacological stimulation ( fig. 6): NPb (Nitroprusside beginning) for [0s, 40s], NPm (Nitroprusside middle) for (40s,80s), NPe (Nitroprusside end) for (80s,120s) and PE (Phenylephrine) for (120s,160s) ( fig. 6). Four normalized vagal indices were defined for each period $\mathrm{P} \in$ (NPb, NPm, NPe, PE) for the HRV indices $E_{H F}$ and the model-based vagal contribution $\mathrm{V}$ :

$$
H F n_{P}=\frac{\int_{P} E_{H F}(t) \mathrm{d} t}{\int_{T_{t o t}} E_{H F}(t) \mathrm{d} t}, V n_{P}=\frac{\int_{P} V(t) \mathrm{d} t}{\int_{T_{t o t}} V(t) \mathrm{d} t}
$$

$H F n_{P}$ and $V n_{P}$ corresponds respectively to the energy of $E_{H F}$ and V, on each period $\mathrm{P}$, normalized with respect to total energy of $E_{H F}$ on the four periods Ptot $=(0,160)(\mathrm{s})$. The definition of theses two indices will allow an accurate comparison between HRV and model-based analysis results.

\section{Results and Discussion}

\subsection{Batch identification of constant parameters}

The model used in this work was implemented using the M2SL simulation library [31]. Results obtained concerning the identification of constant parameters (step 1 of fig. 3) are exposed in table 1. Delays and time constants differ from adult humans concerning both the vagal and sympathetic 
Table 1: Identified values for constant parameters obtained after the batch identification.

\begin{tabular}{lllllll}
\hline$T_{B}(\mathrm{~s})$ & $K_{V}(\mathrm{bpm})$ & $T_{V}(\mathrm{~s})$ & $D_{V}(\mathrm{~s})$ & $K_{S}(\mathrm{bpm})$ & $T_{S}(\mathrm{~s})$ & $D_{S}(\mathrm{~s})$ \\
\hline 0.50 & 76.68 & 0.680 & 0.31 & 98.4 & 11.8 & 4.0 \\
$( \pm 0.05)$ & $( \pm 25.2)$ & $( \pm 0.18)$ & $( \pm 0.23)$ & $( \pm 19.8)$ & $( \pm 1.42)$ & $( \pm 0.20)$
\end{tabular}

systems $[10,8,9]$. Other than the inter-species variability, these differences can be explained by the maturity of the autonomic nervous system, because parameters evolve rapidly during the first days of life. Identification results are in agreement with spectral analysis performed on neonates signals [32].

The results obtained with the batch identification on two representative lambs are shown in fig. 7. The beginning of the RR series corresponds to the nitroprusside injection, and the phenylephrine bolus is injected after 120 seconds. The decrease of AP and RR interval, which can be observed in the first part of the signal, is the consequence of the vasodilation induced by nitroprusside. Then, the RR slowly increases in response to AP augmentation, due to autonomic regulation of hemodynamic variables. Finally, the injection of phenylephrine (at $\mathrm{t}=120$ seconds) induces a vasoconstriction. In both cases, the vagal contribution (V) decreases during nitroprusside injection while sympathetic contribution (S) increases. The phenylephrine induces a rise of vagal and a diminution the sympathetic modulations. This estimation of the vagal and sympathetic contribution is physiologically relevant. However, the batch identification failed to reproduce the whole morphology of the RR response. Theses simulations illustrate the need of more accurate methods in order to estimate the $\mathrm{RR}$ responses to pharmacological stimulation.

\subsection{Recursive identification of time-varying parameters}

Parameter values in Table 1 were used and kept constant during the recursive identification of $M_{V}$ and $M_{S}$. Results obtained with recursive identification are depicted on fig. 8 for the two representative lambs with and without beta-blockers.

Fig. 8 (E,F, G,H) shows the comparison between simulated (black lines) and experimental (red lines) RR intervals after recursive identification. These curves illustrate a satisfactory fit of the model to real data. In order to quantify the difference between experimental and simulated signals, the relative 
Lamb \#1
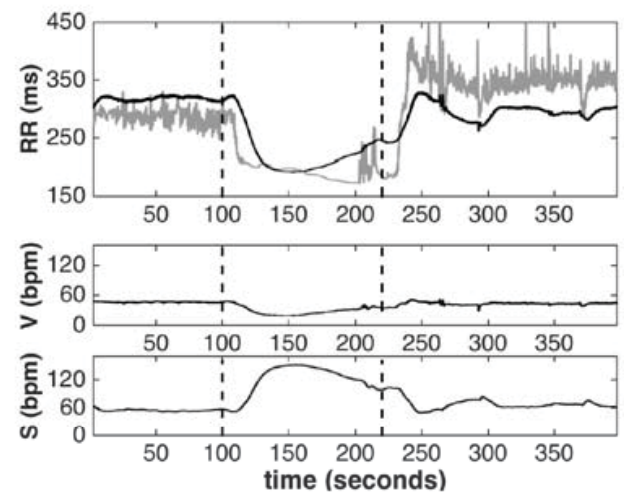

\section{Lamb \#2}
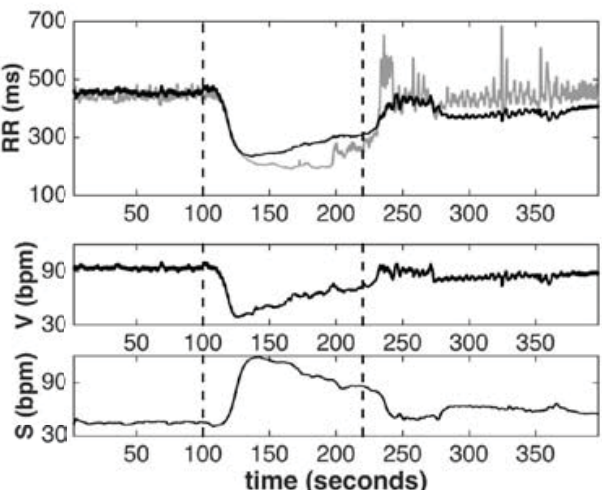

Figure 7: Results obtained with the batch identification. Observed (grey lines) and batch simulated (black lines) RR intervals (ms) acquired from two representative lambs, without sympathetic blockade. Vagal (V) and sympathetic (S) contributions are expressed in bpm as they are added to $H R_{0}$ to obtain current $\mathrm{HR}$

Root Mean Square Error (rRMSE) was computed as

$$
r R M S E=\sqrt{\frac{1}{n} \sum_{i=1}^{n}\left(\frac{R R_{s i m}(i)-R R_{\exp }(i)}{R R_{e x p}(i)}\right)^{2}}
$$

where $\mathrm{n}$ is the number of samples. Indeed, the rRMSE is respectively equal to $0.0030(+/-0.0038)$ and $0.0020(+/-0.0032)$ for the five lambs under normal conditions and under beta-blockers. The comparison of the errors obtained with the batch and recursive identifications is presented in Table 2 . The mean rRMSE, which is equal to 0.0156 and 0.0026 respectively for the batch and recursive identification, illustrates the ability of the recursive identification to provide a more satisfying fit to experimental data.

The mean effects metoprolol were quantified on the baseline period before injection of nitroprusside. Without beta-blockers, the mean AP, HR and inspirations period $\left(T_{i}\right)$ are respectively equal to $359.9(+/-75.1) \mathrm{msec}$, $74.0(+/-11,7) \mathrm{mmHg}$ and $0,57(+/-0,58)$ sec. Under beta-blockers, AP, $\mathrm{HR}$ and $T_{i}$ are respectively equal to $365.0(+/-61,0)$ msec, $365.07(+/-10,7)$ $\mathrm{mmHg}$ and $0.37(+/-0,3)$ sec. These results reflect some variability between 
Lamb \#1
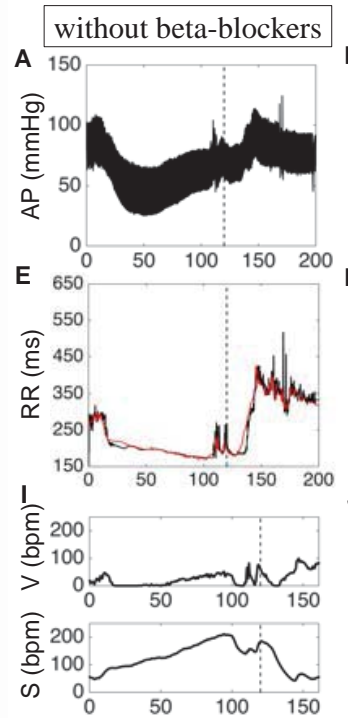

M

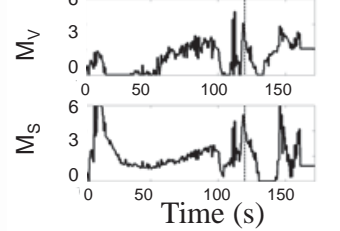

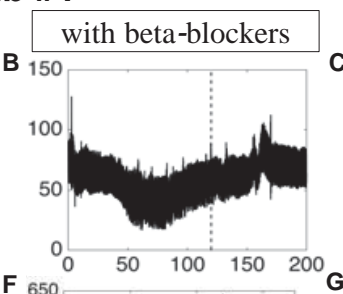

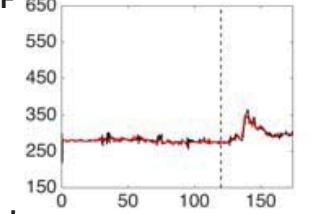

J
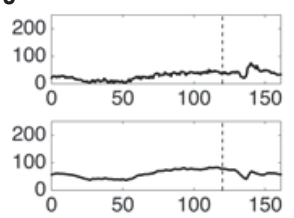

$\mathbf{N}$

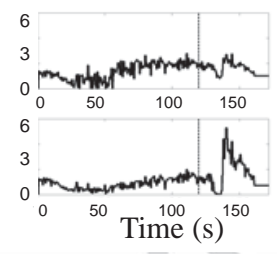

Lamb \#2
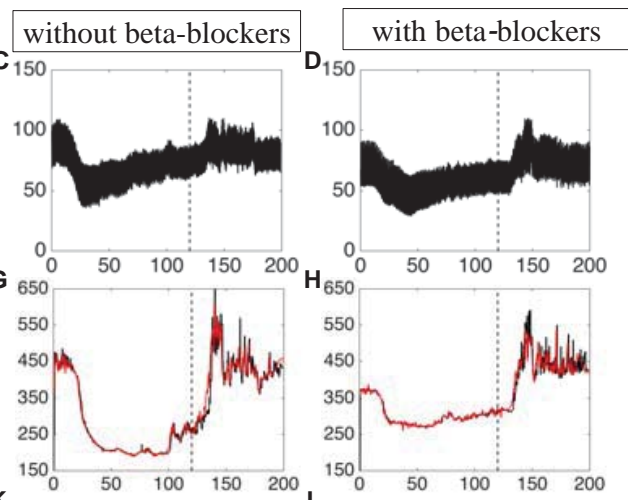

$\mathrm{K}$

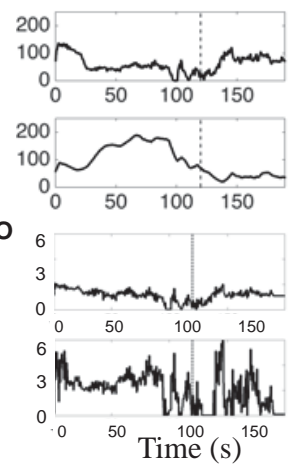

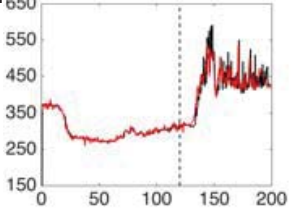

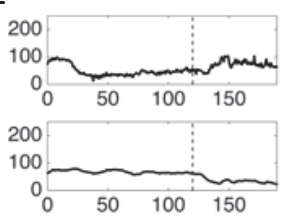

$\mathbf{P}$

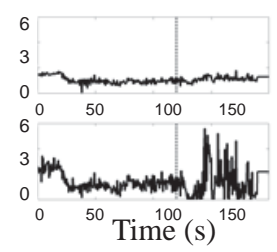

Figure 8: Results obtained for two representative lambs without sympathetic blockade and with beta-blockers. Subcharts (A, B, C, D) present the AP in mmHg. Simulated (red line) and experimental (black line) RR interval (ms) are represented on subchart (D, E, F, G). Contributions of the vagal $(\mathrm{V}, \mathrm{bpm})$ and sympathetic $(\mathrm{S}, \mathrm{bpm})$ activities are illustrated in (I, J, K, L). Contributions of the vagal and sympathetic modulation (respectively $M_{V}$ and $M_{S}$, n.u.) determined by the recursive identification are shown in ( $\left.\mathrm{M}, \mathrm{N}, \mathrm{O}, \mathrm{P}\right)$. The vertical dotted lines (120s) correspond to the injection of phenylephrine. On each diagram, the abscissa is the time expressed in seconds.

Table 2: Comparison between the relative root mean squared error (rRMSE) calculated for each lamb after batch and recursive identification.

\begin{tabular}{lllllll}
\hline rRMSE & Lamb 1 & Lamb 2 & Lamb 3 & Lamb 4 & Lamb 5 & Mean value \\
\hline Batch & 0.0397 & 0.0133 & 0.0031 & 0.0157 & 0.0063 & $\mathbf{0 . 0 1 5 6}$ \\
Identification & & & & & &
\end{tabular}

$\begin{array}{lllllll}\text { Recursive } & 0.0023 & 0.0024 & 0.0002 & 0.0077 & 0.0004 & \mathbf{0 . 0 0 2 6}\end{array}$


sheep responses to metoprolol. Although the experimental conditions were standardized (drug doses, state of alertness,...), the experiments (with and without beta-blockers) lasted for 2 days and the uncontrolled variations of environnemental conditions could explain the observed variability.

The estimated contributions of vagal and sympathetic pathways, which both describe the tonic and reflex feature of autonomic modulation, are shown in fig. 8 (I, K) with no sympathetic blockade. During the first 100 seconds, these signals are characterized by a decrease of vagal modulation and an increase of sympathetic modulation. Then, the parasympathetic contribution begins to rise and the sympathetic contribution falls because AP stabilizes. After the injection of phenylephrine occurring at 120 seconds, the vagal modulation suddenly rises and, then, is maintained while sympathetic activities slowly decrease after the injection.

The curves, shown in fig. $8(\mathrm{~J}, \mathrm{~L})$, depicts the contributions of vagal and sympathetic pathways with beta-blockers. Although the injection of nitroprusside is performed at the beginning $(\mathrm{t}=0 \mathrm{~s})$, vagal and sympathetic contributions are relatively stable until the injection of phenylephrine. After 120 seconds, parasympathetic modulation shows a little increase and then stabilizes. The sympathetic activities remain stable because beta-blockers block the action of the sympathetic nervous system. Model-based estimations of the dynamics of vagal and sympathetic activities illustrate different behaviors in the absence of a sympathetic blockade drug and with beta-blockers. In the first case, the baroreflex modulation allows for the stabilization of AP, while this stabilization is not observed while using beta-blockers. The residual sympathetic contribution to HR after beta-blockade could be explained by the intrinsic sympathomimetic activity of metoprolol. These results show how the proposed approach may be used to estimate the individual dynamic responses of the sympathetic and parasympathetic systems during autonomic maneuvers.

fig. 8 (M,N,O,P) also illustrates the evolution of the identified variables $M_{V}(\mathrm{t})$ and $M_{S}(\mathrm{t})$. These two time-varying variables reflect the part of the neural activity that is not explicitly represented within the model as functions of AP or respiration: central modulation, impact of the closed-loop structure of the baroreflex or the influence of drug administration. Under beta-blockers, a diminution of the sympathetic modulation $(\mathrm{S})$ is associated with significant variations of $M_{S}$ after the phenylephrine administration. In fact, the variations of $M_{S}$ balance the neural activity coming from the baroreceptors that directly depends on the arterial pressure. The interpretation of 
$M_{V}(\mathrm{t})$ and $M_{S}(\mathrm{t})$ are quite uneasy because they aggregate several physiological phenomena. However, their influences on vagal and sympathetic pathways are essential to reproduce the response of HR in response to arterial pressure variations. In the following part of the article, we will focus on the vagal and sympathetic contributions (V and $\mathrm{S}$ ).

\subsection{Comparison with classical HRV indexes}

The estimations of the sympathetic and vagal pathways, presented in fig. 8 (I,J,K,L), seem to be consistent with physiological knowledge. However, the proposed approach should be compared to other well recognized methods. Frequential methods are known as references for the HRV analysis. Because of the non-stationnarity of the signals under study, it was necessary to use time-frequency methods, as explained in section 2.4. As the HF component of the RR series is associated with cardiac efferent parasympathetic modulation, the evolution of HF component is compared to the model-based estimation of vagal modulation.

The boxplot, exposed in fig. 9, illustrates that indices obtained with HRV and model-based analysis have the same evolution during the pharmacological stimulation. This comparison highlights the consistency of the estimation of the vagal pathway using the modeling approach. However, it is important to understand that our goal is not to reach a maximal similarity. Our hypothesis is that the model-based approach provides a better estimation of the vagal modulation, since it takes into account both the high-frequency and the low-frequency components of the parasympathetic modulation.

\section{Conclusion}

In this paper, a model-based approach was used to estimate the vagal and sympathetic contributions to HR modulation during pharmacological stimulations. The main originality is to propose a recursive identification of the parasympathetic and sympathetic modulations. This strategy of identification improves the ability of the baroreflex model to reproduce experimental signals, providing time-varying estimations of the individual dynamics of the vagal and sympathetic activities that were consistent with physiological knowledge and traditional HRV markers. The advantage of the proposed model-based approach is to bring information on the evolution of sympathovagal balance in non-stationary conditions and to include physiological knowledge on the data processing. 
Without Beta-Blockers

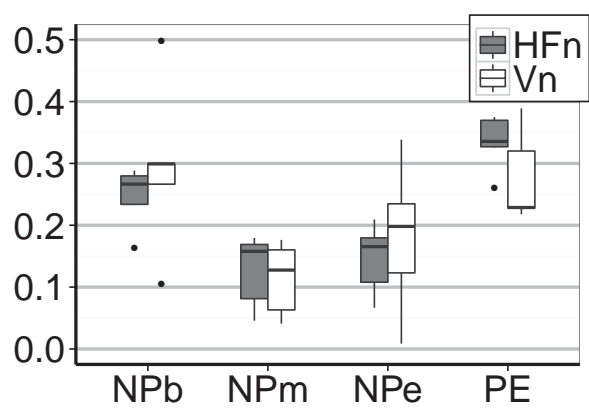

With Beta-Blockers

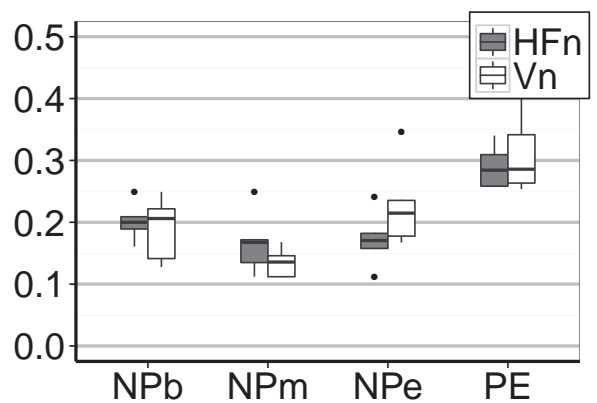

Figure 9: Boxplot of indices obtained from classical HRV analysis (HFn) and with the model-based approach $(\mathrm{Vn})$ for the five lambs. Four periods of 40 seconds were defined in order to capture the variations of $\mathrm{HF}$ during the pharmacological stimulation: $\mathrm{NPb}$ (Nitroprusside beginning) for (0s,40s), NPm (Nitroprusside middle) for $(40 \mathrm{~s}, 80 \mathrm{~s})$, NPe (Nitroprusside end) for (80s,120s) and PE (Phenylephrine) for (120s,160s).

The proposed model-based approach could be used to analyze time-varying vagal and sympathetic modulations during various non-stationary conditions associated with pediatric period. Future works will focus on the analysis of the autonomic response to laryngeal chemoreflexes (LCR). Our previous results [33], based on classical HRV analysis, highlights a joint increase of sympathetic and parasympathetic tones after laryngeal stimulation in term lambs during quiet sleep. Although the interactions in sympathetic and vagal HR controls is not directly represented in the model, the recursive identification algorithm could estimate sympathovagal coactivation during LCR by the mean of time-varying variables $M_{V}$ and $M_{S}$. The baroreflex model could also be improved by including: i) a better representation of the cardiorespiratory interactions, based on our previous studies on ventilation [20], ii) the introduction of an accurate model of circulation, which is associated with an increased number of parameters that will require the use of sensitivity methods before the identification $[34,23]$. The results presented in this paper are encouraging for the use of this model-based approach in computer-aided diagnosis, and for testing different therapeutic scenarios with a patient-specific model, especially in a pediatric context. 


\section{Acknowledgments}

This study was supported by the Canada Research Chair in Neonatal Respiratory Physiology allocated to J-P Praud.

\section{References}

[1] M. Orini, R. Bailon, L. Mainardi, P. Laguna, P. Flandrin, Characterization of the dynamic interactions between cardiovascular signals by time?frequency coherence, IEEE Trans. Biomed. Eng 59 (3) (2012) 66373 .

[2] R. Vetter, N. Virag, J. Vesin, P. Celka, U. Scherrer, Observer of autonomic cardiac outflow based on blind source separation of ecg parameters, EEE Trans Biomed Eng 47 (5) (2000) 578-82.

[3] A. Kachenoura, G. Carrault, L. Senhadji, A. Hernandez, Extraction des dynamiques du systëme nerveux autonome : Une approche basèe sur la sèparation aveugle de sources, Colloque GRETSI.

[4] D. Eckberg, Sympathovagal balance: a critical appraisal, Circulation 96 (9) (1997) 3224-32.

[5] G. Baselli, S. Cerutti, F. Badilini, L. Biancardi, A. Porta, M. Pagani, F. Lombardi, O. Rimoldi, R. Furlan, A. Malliani, Model for the assessment of heart period and arterial pressure variability interactions and of respiration influences, Med Biol Eng Comput 32 (2) (1994) 143-52.

[6] A. Porta, G. Baselli, O. Rimoldi, A. Malliani, M. Pagani, Assessing baroreflex gain from spontaneous variability in conscious dogs: role of causality and respiration, Am J Physiol Heart Circ Physiol. 279 (5) (2000) H2558-67.

[7] A. Porta, P. Castiglioni, M. Di Rienzo, T. Bassani, V. Bari, L. Faes, G. Nollo, A. Cividjan, L. Quintin, Cardiovascular control and time domain granger causality: insights from selective autonomic blockade., Philos Trans A Math Phys Eng Sci 371 (2013) 20120161.

[8] M. Ursino, E. Magosso, Role of short-term cardiovascular regulation in heart period variability: a modeling study, Am J Physiol Heart Circ Physiol 284 (4) (2003) H1479-93. 
[9] A. Van Roon, L. Mulder, M. Althaus, M. G, Introducing a baroreflex model for studying cardiovascular effects of mental workload, Psychophysiology 41 (6) (2004) 961-81.

[10] W. Wesselink, D. Holsheimer, H. Boom, A model of the electrical behaviour of myelinated sensory nerve fibres based on human data, Medical and Biological Engineering and Computing 37 (2) (1999) 228-35.

[11] K. Lu, J. J. Clark, F. Ghorbel, D. Ware, A. Bidani, A human cardiopulmonary system model applied to the analysis of the valsalva maneuver, Am J Physiol Heart Circ Physiol 281 (6) (2001) H2661-79.

[12] T. Kawada, K. Uemura, S. Shimizu, A. Kamiya, M. Turner, M. Mizuno, K. Sunagawa, M. Sugimachi, Consideration on parameter determination of a new model describing dynamic vagal heart rate control in rats, Annual International Conference of the IEEE Engineering in Medicine and Biology Society (EMBC) (2012) 3809-3812.

[13] J. Ottesen, Nonlinearity of baroreceptor nerves., Surv. Math. Ind. (1997) 187201.

[14] J. Ottesen, M. Olufsen, Functionality of the baroreceptor nerves in heart rate regulation., Comput Methods Programs Biomed. 101 (2) (2011) 208-19.

[15] T. Heldt, E. Shim, R. Kamm, M. RG, Computational modeling of cardiovascular response to orthostatic stress, J Appl Physiol (1985). 2002 Mar;92(3):1239-54. 92 (3) (2002) 1239-54.

[16] M. Olufsen, H. Tran, J. Ottesen, L. Lipsitz, V. Novak, Modeling baroreflex regulation of heart rate during orthostatic stress, Am J Physiol Regul Integr Comp Physiol 291 (5) (2006) R1355-68.

[17] V. Le Rolle, A. Hernndez, P.-Y. Richard, C. G., An autonomic nervous system model applied to the analysis of orthostatic tests, Modelling and Simulation in Engineering (2008) 427926.

[18] V. Le Rolle, A. Hernandez, P. Richard, J. Buisson, G. Carrault, A bond graph model of the cardiovascular system, Acta Biotheor. 53 (4) (2005) 295-312. 
[19] C. Duvareille, M. Lafrance, N. Samson, M. St-Hilaire, P. Pladys, P. Micheau, V. Bournival, C. Langlois, P. JP., Effects of hypoxia and hypercapnia on nonnutritive swallowing in newborn lambs, J Appl Physiol 103 (4) (2007) 1180-8.

[20] V. Le Rolle, D. Ojeda, A. BeuchÈe, J. Praud, P. Pladys, A. Hernndez, A model-based approach for the evaluation of vagal and sympathetic activities in a newborn lamb, Conf Proc IEEE Eng Med Biol Soc (2013) $3881-4$.

[21] N. Montano, E. Tobaldini, A. Porta, The autonomic nervous system, Stress Challenges and Immunity in Space (2012) 71-86.

[22] K. T. Sunagawa K, N. T., Dynamic nonlinear vago-sympathetic interaction regulating heart rate., Heart Vessels 13 (1998) 157-174.

[23] V. Le Rolle, D. Ojeda, A. Hernandez, Embedding a cardiac pulsatile model into an integrated model of the cardiovascular regulation for heart failure followup, IEEE Trans Biomed Eng 58 (10) (2011) 2982-6.

[24] Z. Michalewicz, Genetic algorithms + data structures $=$ evolution programs., ed. Springer-Verlag, New York.

[25] T. of the European Society of Cardiology, the North American Society of Pacing, Electrophysiology, Heart rate variability: standards of measurement, physiological interpretation and clinical use, Circulation 93 (5) (1996) 1043-65.

[26] A. Beuchee, A. Hernandez, C. Duvareille, D. Daniel, N. Samson, P. Pladys, J. Praud, Influence of hypoxia and hypercapnia on sleep statedependent heart rate variability behavior in newborn lambs, Sleep 35 (11) (2012) 1541-9.

[27] S. Akselrod, D. Gordon, F. Ubel, D. Shannon, A. Berger, C. RJ, Power spectrum analysis of heart rate fluctuation: a quantitative probe of beatto-beat cardiovascular control, Science 213 (4504) (1981) 220-2.

[28] M. Appel, R. Berger, J. Saul, J. Smith, R. Cohen, Beat to beat variability in cardiovascular variables: noise or music?, J Am Coll Cardiol 14 (5) (1989) 1139-48. 
[29] B. GE, The lf/hf ratio does not accurately measure cardiac sympathovagal balance, Front Physiol (2013) 4:26.

[30] A. Costa, G. Boudreau-Bartels, Design of time-frequency representations using a multiform, tiltable exponential kernel, IEEE Trans. Signal Process 43 (1995) $2283-2301$.

[31] A. Hernandez, V. Le Rolle, A. Defontaine, G. Carrault, A multiformalism and multiresolution modelling environment: application to the cardiovascular system and its regulation, Philosophical Transactions Mathematical Physical Engineering Sciences 367 (2009) 4923-4940.

[32] P. Andriessen, A. Koolen, R. Berendsen, P. Wijn, E. ten Broeke, S. Oei, C. Blanco, Cardiovascular fluctuations and transfer function analysis in stable preterm infants, Pediatr Res. 53 (1) (2003) 89-97.

[33] P. dynamic changes in autonomic heart rate modulation induced by acid laryngeal stimulation in non-sedated lambs, A beuchee and e nsegbe and $\mathrm{m}$ st hilaire and g carrault and b branger and p pladys and j-p praud, Neonatology 91 (2) (2007) 83-91.

[34] A. Hernandez, V. Le Rolle, D. Ojeda, P. Baconnier, J. Fontecave-Jallon, F. Guillaud, T. Grosse, R. Moss, P. Hannaert, T. S.R, Integration of detailed modules in a core model of body fluid homeostasis and blood pressure règulation, Progress in Biophysics and Molecular Biology 107 (2011) 169:182 